\title{
A generalization of Mason's theorem for four polynomials
}

\author{
M. Bayat and H. Teimoori*
}

\begin{abstract}
M. Bayat studied mathematics at the Imam Khomeiny International University of Qazvin in Iran. In 1996, he received his M.S. in mathematics from the Institute for Advanced Studies in Basic Sciences of Zanjan in Iran, where he is a research assistant since 1999. His research interests include combinatorics, number theory, commutative algebra, ordinary differential equations and mathematics education.

H. Teimoori studied mechanical engineering at the AmirKabeer University of Technology of Tehran in Iran. In 1999, he received his M.S. in mathematics from the Institute for Advanced Studies in Basic Sciences at Zanjan in Iran, where he is now a research assistant. His research interests include combinatorics, number theory and mathematics education.
\end{abstract}

In 1981, W. Stothérs [4] discovered a new, very interesting fact about polynomials. This theorem was not being paid attention by mathematicians until R.C. Mason [2] rediscovered this theorem in 1983, and Fermat's last theorem for non-constant polynomials could be proved in a simple way by means of this theorem. Of course, Fermat's last theorem for polynomials had already been proved before using algebraic geometric tools.

Vor nunmehr fast zehn Jahren wurde die Vermutung von Fermat durch A. Wiles bewiesen. Über diese spektakuläre Entdeckung wurde in dieser Zeitschrift mehrfach berichtet. Die analoge Vermutung für Polynome war bereits lange zuvor bewiesen worden; ein eleganter Beweis stammt von R.C. Mason aus dem Jahr 1983, in dem er einen bereits 1981 von W. Stothérs gefundenen, aber unbeachtet gebliebenen Satz wiederentdeckte. Auch darüber und N. Snyders vereinfachenden Beweis wurde hier berichtet. In der vorliegenden Arbeit beweisen die Autoren in Verallgemeinerung dazu, dass es keine paarweise teilerfremden, nicht-konstanten Polynome $a, b, c, d \in \mathbb{C}[t]$ gibt, die der Gleichung

$$
a(t)^{n_{1}}+b(t)^{n_{2}}+c(t)^{n_{3}}=d(t)^{n_{4}}
$$

genügen, sobald $\min \left\{n_{1}, n_{2}, n_{3}, n_{4}\right\} \geq 8$ gilt.

*) The authors are supported in part by the Institute for Advanced Studies in Basic Sciences, Zanjan, IRAN. 
First of all, let us introduce some elementary tools that will be needed later to prove our theorem.

We consider polynomials with complex numbers as coefficients. The set of all such polynomials in a variable $t$ is denoted by $\mathbb{C}[t]$; if $f \in \mathbb{C}[t], f \neq 0$, we write

$$
f(t)=c \prod_{i=1}^{r}\left(t-\alpha_{i}\right)^{m_{i}}
$$

where $\alpha_{1}, \alpha_{2}, \ldots, \alpha_{r}$ are the distinct roots of $f$, and $c$ is a constant, $c \neq 0$. The integers $m_{i}(i=1,2, \ldots, r)$ are the multiplicities of the roots, and the degree of the polynomial $f$ is

$$
\operatorname{deg}(f)=m_{1}+\cdots+m_{r}
$$

The number of (distinct) roots of $f$ will be denoted by $n_{0}(f)$, so by definition

$$
n_{0}(f)=r
$$

It is obvious that $\operatorname{deg}(f)$ can be very large, but $n_{0}(f)$ may be small. For instance, $f(t)=(t-\alpha)^{10000}$ has degree 10000 , but $n_{0}(f)=1$. If $f, g$ are two non-zero polynomials, then in general

$$
n_{0}(f g) \leq n_{0}(f)+n_{0}(g)
$$

In addition, if $f, g$ are relatively prime, then we actually have the equality

$$
n_{0}(f g)=n_{0}(f)+n_{0}(g)
$$

Taking into account the above notations, we can now state Mason's theorem.

Theorem 1 (Mason's theorem) Let $f, g, h \in \mathbb{C}[t]$ be non-constant relatively prime polynomials satisfying $f+g=h$. Then, we have

$$
\max \{\operatorname{deg}(f), \operatorname{deg}(g), \operatorname{deg}(h)\} \leq n_{0}(f g h)-1
$$

It can be easily seen that the equality is true by considering the following example:

Example:

$$
\begin{aligned}
& f(t)=\left(t^{3}+4 t^{2}+10 t+6\right)^{3} \\
& g(t)=t\left(t^{4}+6 t^{3}+21 t^{2}+35 t+\frac{63}{2}\right)^{2} \\
& h(t)=27 t^{2}+\frac{351}{4} t+216
\end{aligned}
$$

Mason's theorem has been proved in [1,3] using logarithmic derivatives and divisibility properties. Here, we generalize this theorem to four polynomials using the same argument as in [3]. 
Theorem 2 Let $f, g, h, k \in \mathbb{C}[t]$ be non-constant relatively prime polynomials satisfying $f+g+h=k$. Then, we have

$$
\max \{\operatorname{deg}(f), \operatorname{deg}(g), \operatorname{deg}(h), \operatorname{deg}(k)\} \leq 2 n_{0}(f g h k)-3 .
$$

Proof. Without loss of generality, we may assume that $\operatorname{deg}(f)$ is maximal. Thus, it is necessary to show that

$$
\operatorname{deg}(f) \leq 2 n_{0}(f g h k)-3 .
$$

To do this, we divide the equation $f+g+h=k$ by $k$, and obtain

$$
\frac{f}{k}+\frac{g}{k}+\frac{h}{k}=1 \text {. }
$$

Put $R=\frac{f}{k}, S=\frac{g}{k}$ and $T=\frac{h}{k}$. Then $R+S+T=1$. Now, by taking derivatives on both sides of the above equation and dividing by $T^{\prime}$, we get

$$
\frac{R^{\prime}}{T^{\prime}}+\frac{S^{\prime}}{T^{\prime}}=-1
$$

Now, repeating the above argument, putting $E=\frac{R^{\prime}}{T^{\prime}}, F=\frac{S^{\prime}}{T^{\prime}}$ and taking derivatives, we get the new equation $E^{\prime}+F^{\prime}=0$, which we rewrite in the form

$$
\frac{E^{\prime}}{E} E+\frac{F^{\prime}}{F} F=0
$$

or, equivalently,

$$
\frac{E}{F}=-\frac{F^{\prime} / F}{E^{\prime} / E}
$$

Consider the quotient $f / g$. With our notation and the above equation, we obtain

$$
\frac{f}{g}=\frac{\frac{g^{\prime \prime}}{g} \times \frac{h^{\prime}}{h}-\frac{g^{\prime \prime}}{g} \times \frac{k^{\prime}}{k}-\frac{k^{\prime \prime}}{k} \times \frac{h^{\prime}}{h}-\frac{h^{\prime \prime}}{h} \times \frac{g^{\prime}}{g}+\frac{h^{\prime \prime}}{h} \times \frac{k^{\prime}}{k}+\frac{k^{\prime \prime}}{k} \times \frac{g^{\prime}}{g}}{\frac{h^{\prime}}{h}-\frac{f^{\prime \prime}}{f} \times \frac{k^{\prime}}{k}-\frac{k^{\prime \prime}}{k} \times \frac{h^{\prime}}{h}-\frac{h^{\prime \prime}}{h} \times \frac{f^{\prime}}{f}+\frac{h^{\prime \prime}}{h} \times \frac{k^{\prime}}{k}+\frac{k^{\prime \prime}}{k} \times \frac{f^{\prime}}{f}}
$$

or, equivalently,

$$
\frac{f}{g}=\left|\begin{array}{ccc}
1 & 1 & 1 \\
\frac{g^{\prime}}{g} & \frac{h^{\prime}}{h} & \frac{k^{\prime}}{k} \\
\frac{g^{\prime \prime}}{g} & \frac{h^{\prime \prime}}{h} & \frac{k^{\prime \prime}}{k}
\end{array}\right| \cdot\left|\begin{array}{ccc}
1 & 1 & 1 \\
\frac{f^{\prime}}{f} & \frac{h^{\prime}}{h} & \frac{k^{\prime}}{k} \\
\frac{f^{\prime \prime}}{f} & \frac{h^{\prime \prime}}{h} & \frac{k^{\prime \prime}}{k}
\end{array}\right|^{-1} .
$$

If we denote the right-hand side by $P / Q$, the following equality results:

$$
f \cdot Q=g \cdot P .
$$

First, we rewrite the equality $f+g+h=k$ as $g+h-k=-f$. Now, we distinguish two cases: 
Case I. Suppose $g, h$ and $k$ are linearly independent over $\mathbb{C}$. Therefore, the following Wronskian is a non-zero polynomial:

$$
\left|\begin{array}{lll}
g(x) & h(x) & k(x) \\
g^{\prime}(x) & h^{\prime}(x) & k^{\prime}(x) \\
g^{\prime \prime}(x) & h^{\prime \prime}(x) & k^{\prime \prime}(x)
\end{array}\right| \neq 0
$$

Since the above polynomial and $g, h, k$ have a finite number of roots, there is a number $\alpha \in \mathbb{C}$, such that

$$
0 \neq\left|\begin{array}{ccc}
g(\alpha) & h(\alpha) & k(\alpha) \\
g^{\prime}(\alpha) & h^{\prime}(\alpha) & k^{\prime}(\alpha) \\
g^{\prime \prime}(\alpha) & h^{\prime \prime}(\alpha) & k^{\prime \prime}(\alpha)
\end{array}\right|=g(\alpha) h(\alpha) k(\alpha)\left|\begin{array}{ccc}
1 & 1 & 1 \\
\frac{g^{\prime}(\alpha)}{g(\alpha)} & \frac{h^{\prime}(\alpha)}{h(\alpha)} & \frac{k^{\prime}(\alpha)}{k(\alpha)} \\
\frac{g^{\prime \prime}(\alpha)}{g(\alpha)} & \frac{h^{\prime \prime}(\alpha)}{h(\alpha)} & \frac{k^{\prime \prime}(\alpha)}{k(\alpha)}
\end{array}\right|
$$

and, consequently,

$$
\left|\begin{array}{ccc}
1 & 1 & 1 \\
\frac{g^{\prime}(\alpha)}{g(\alpha)} & \frac{h^{\prime}(\alpha)}{h(\alpha)} & \frac{k^{\prime}(\alpha)}{k(\alpha)} \\
\frac{g^{\prime \prime}(\alpha)}{g(\alpha)} & \frac{h^{\prime \prime}(\alpha)}{h(\alpha)} & \frac{k^{\prime \prime}(\alpha)}{k(\alpha)}
\end{array}\right| \neq 0
$$

Thus, the rational function $P$ and consequently $Q$, is a non-zero polynomial. Now, we suppose

$$
\begin{array}{ll}
f(t)=c_{1} \prod\left(t-\alpha_{i}\right)^{m_{i}}, & g(t)=c_{2} \prod\left(t-\beta_{j}\right)^{n_{j}}, \\
h(t)=c_{3} \prod\left(t-\gamma_{s}\right)^{p_{s}}, & k(t)=c_{4} \prod\left(t-\delta_{l}\right)^{q_{l}} .
\end{array}
$$

Taking logarithmic derivatives of $f(t), g(t), h(t), k(t)$, we get respectively:

$$
\begin{array}{rlrl}
\frac{f^{\prime}}{f} & =\sum \frac{m_{i}}{\left(t-\alpha_{i}\right)}, & \frac{f^{\prime \prime}}{f}=\left(\sum \frac{m_{i}}{\left(t-\alpha_{i}\right)}\right)^{2}-\sum \frac{m_{i}}{\left(t-\alpha_{i}\right)^{2}}, \\
\frac{g^{\prime}}{g}=\sum \frac{n_{j}}{\left(t-\beta_{j}\right)}, & \frac{g^{\prime \prime}}{g}=\left(\sum \frac{n_{j}}{\left(t-\beta_{j}\right)}\right)^{2}-\sum \frac{n_{j}}{\left(t-\beta_{j}\right)^{2}}, \\
\frac{h^{\prime}}{h}=\sum \frac{p_{s}}{\left(t-\gamma_{s}\right)}, & \frac{h^{\prime \prime}}{h}=\left(\sum \frac{p_{s}}{\left(t-\gamma_{s}\right)}\right)^{2}-\sum \frac{p_{s}}{\left(t-\gamma_{s}\right)^{2}}, \\
\frac{k^{\prime}}{k}=\sum \frac{q_{l}}{\left(t-\delta_{l}\right)}, & \frac{k^{\prime \prime}}{k}=\left(\sum \frac{q_{l}}{\left(t-\delta_{l}\right)}\right)^{2}-\sum \frac{q_{l}}{\left(t-\delta_{l}\right)^{2}} .
\end{array}
$$

For the above rational functions, the quantity

$$
D(t)=\prod\left(t-\alpha_{i}\right)^{2} \prod\left(t-\beta_{j}\right)^{2} \prod\left(t-\gamma_{s}\right)^{2} \prod\left(t-\delta_{l}\right)^{2}
$$

is a common denominator; obviously, we have $\operatorname{deg}(D(t))=2 n_{0}(f g h k)$. We multiply both sides of (3) by $D(t)$. It is clear that both sides are polynomials. We then obtain

$$
f \cdot Q \cdot D(t)=g \cdot P \cdot D(t),
$$


or, equivalently,

$$
f \mid g \cdot P \cdot D(t) .
$$

Since $(f, g)=1$, we find

$$
f \mid P \cdot D(t) .
$$

Now, considering (1), we get

$$
\begin{aligned}
& \operatorname{deg}(f) \leq \operatorname{deg}(P \cdot D(t)) \\
& \leq \max \left\{\operatorname{deg}\left(D(t) \cdot \frac{g^{\prime \prime}}{g} \cdot \frac{h^{\prime}}{h}\right), \operatorname{deg}\left(D(t) \cdot \frac{g^{\prime \prime}}{g} \cdot \frac{k^{\prime}}{k}\right), \ldots, \operatorname{deg}\left(D(t) \cdot \frac{k^{\prime \prime}}{k} \cdot \frac{g^{\prime}}{g}\right)\right\} .
\end{aligned}
$$

Since

$$
\operatorname{deg}(D(t))=2 n_{0}(f g h k),
$$

we conclude that

$$
\operatorname{deg}(f) \leq 2 n_{0}(f g h k)-3
$$

Case II. Assume that $g, h$ and $k$ are linearly dependent over $\mathbb{C}$, and $g$ is a linear combination of $h, k$ over $\mathbb{C}$. Therefore, there are $\lambda, \mu \in \mathbb{C}$ satisfying

$$
g=\lambda h+\mu k
$$

Since $g, h$ and $k$ are relatively prime in pairs, then $\lambda \neq 0$ and $\mu \neq 0$. By using Theorem 1 and $n_{0}(g h k)-1<n_{0}(f g h k)-1$, we obtain

$$
\max \{\operatorname{deg}(g), \operatorname{deg}(h), \operatorname{deg}(k)\} \leq n_{0}(f g h k)-1
$$

Since, $-f=g+h-k$ and $n_{0}(f g h k)-1 \leq 2 n_{0}(f g h k)-3$, we get

$$
\max \{\operatorname{deg}(f), \operatorname{deg}(g), \operatorname{deg}(h), \operatorname{deg}(k)\} \leq 2 n_{0}(f g h k)-3 .
$$

Remark 3 If $f, g, h, k$ are linearly dependent, then

$$
\max \{\operatorname{deg}(f), \operatorname{deg}(g), \operatorname{deg}(h), \operatorname{deg}(k)\} \leq 2 n_{0}(f g h k)-5 .
$$

\section{Corollary 4}

$$
\operatorname{deg}(f g h k) \leq 8 n_{0}(f g h k)-12
$$

As an application of our main theorem, we prove that a generalized version of Fermat's last theorem for polynomials holds true under certain conditions.

Theorem 5 Let $n=\min \left\{n_{1}, n_{2}, n_{3}, n_{4}\right\}$ be an integer $\geq 8$. Then, there is no solution of the equation

$$
a(t)^{n_{1}}+b(t)^{n_{2}}+c(t)^{n_{3}}=d(t)^{n_{4}}
$$

with non-constant relatively prime polynomials $a, b, c, d \in \mathbb{C}[t]$. 
Proof. Let $f(t)=a(t)^{n_{1}}, g(t)=b(t)^{n_{2}}, h(t)=c(t)^{n_{3}}$ and $k(t)=d(t)^{n_{4}}$. Then, our main theorem yields

$$
\operatorname{deg}\left(a^{n_{1}}\right) \leq 2 n_{0}\left(a^{n_{1}} b^{n_{2}} c^{n_{3}} d^{n_{4}}\right)-3 .
$$

However $\operatorname{deg}\left(a^{n_{1}}\right)=n_{1} \operatorname{deg}(a)$ and $n_{0}\left(a^{n_{1}}\right) \leq \operatorname{deg}(a)$. Hence, we have

$$
n \operatorname{deg}(a) \leq n_{1} \operatorname{deg}(a) \leq 2(\operatorname{deg}(a)+\operatorname{deg}(b)+\operatorname{deg}(c)+\operatorname{deg}(d))-3 .
$$

Similarly, we obtain analogous inequalities for $b, c$ and $d$, i.e.,

$$
\begin{aligned}
& n \operatorname{deg}(b) \leq 2(\operatorname{deg}(a)+\operatorname{deg}(b)+\operatorname{deg}(c)+\operatorname{deg}(d))-3, \\
& n \operatorname{deg}(c) \leq 2(\operatorname{deg}(a)+\operatorname{deg}(b)+\operatorname{deg}(c)+\operatorname{deg}(d))-3, \\
& n \operatorname{deg}(d) \leq 2(\operatorname{deg}(a)+\operatorname{deg}(b)+\operatorname{deg}(c)+\operatorname{deg}(d))-3 .
\end{aligned}
$$

Adding the three inequalities yields

$$
n \operatorname{deg}(a b c d) \leq 8 \operatorname{deg}(a b c d)-12,
$$

or

$$
(n-8) \operatorname{deg}(a b c d) \leq-12 .
$$

The last inequality implies $n-8<0$, or $n<8$, which contradicts our theorem's hypothesis. Thus the proof is complete.

\section{References}

[1] Lang, S.: Math Talks for Undergraduates. Spinger Verlag, 1999.

[2] Mason, R.C.: Diophantine Equations Over Function Fields. London Math. Soc. Lecture Note Series, Vol. 96, Cambridge University Press, 1984.

[3] Snyder, N.: An Alternate Proof of Mason's Theorem. Elem. Math. 55 (2000) 3, 93-94.

[4] Stothérs, W.: Polynomial identities and hauptmoduln. Quart. Math. Oxford 32 (1981) 2, 349-370.

M. Bayat

Institute for Advanced Studies

in Basic Sciences

P.O.Box 45195-159

Zanjan, Iran

e-mail Bayat@iasbs.ac.ir

H. Teimoori

Institute for Advanced Studies

in Basic Sciences

P.O.Box 45195-159

Zanjan, Iran

e-mail Teimoori@iasbs.ac.ir 